\title{
2D Angularly Dependent Array Error Calibration for 1D Array via Neural Network with Local Manifold Interpolation
}

\author{
Yujian PAN ${ }^{1,2,3}$, Sreeraj RAJENDRAN ${ }^{3}$, Sofie POLLIN ${ }^{3}$ \\ ${ }^{1}$ School of Electronics and Information, Hangzhou Dianzi University, 310018 Hangzhou, China \\ ${ }^{2}$ State Key Laboratory of Millimeter Waves, Southeast University, 210096 Nanjing, China \\ ${ }^{3}$ Dept. of Electrical Engineering, KU Leuven, 3001 Leuven, Belgium \\ pyj@hdu.edu.cn, \{sreeraj.rajendran, sofie.pollin\}@esat.kuleuven.be \\ Submitted January 18, 2021 / Accepted July 2, 2021
}

\begin{abstract}
The calibration of the angularly dependent array error is a challenging task for signal processing. In this paper, we propose a neural network (NN)-based twodimensional (2D) calibration method for a linear array. Firstly, the array steering vectors are measured on an azimuth grid at different elevations in an anechoic chamber, and the off-grid steering vectors are derived by the proposed local manifold interpolation (LMI) technique to reduce the risk of model overfitting. Then, the phase differences are extracted to form the features of the training data. At last, noise is added to the training data to enable the NN model to generalize well to the noisy data. The proposed method is evaluated by the indoor and outdoor measured data from a $77 \mathrm{GHz}$ automotive radar and is compared with the conventional signal processing-based methods. The evaluation results show that a single NN model trained at the lowest signal-to-noise ratio (SNR) outperforms conventional methods by at least $55 \%$ on average over the entire SNR range and gives close performance to the perfect array without array error at low to medium SNR.
\end{abstract}

\section{Keywords}

Angularly dependent array error, automotive radar, direction-of-arrival (DOA) estimation, local manifold interpolation, neural network

\section{Introduction}

The array signal processing is a technique widely applied in civilian and military fields, such as radar, sonar, communication, and seismology [1]. For theoretical research, the responses of the array sensors are assumed to be the same so that the array steering vector has a simple analytical expression that depends only on the direction of arrival (DOA) of the signal when the wavelength and array geometry are fixed.
However, the above assumption usually is not true. Due to the production errors, the sensors are not exactly the same, which leads to the gain/phase error. Besides, for the avoidance of spatial ambiguity, the sensors cannot be placed too sparsely, which results in mutual coupling between sensors. Moreover, the fabrication error and difference between sensor's phase center and physical position will cause the sensor position error. For the simplification of the array error calibration, these array errors are assumed to be independent of the DOA. Thus many online calibration methods that jointly estimate the array errors and source parameters can be proposed [2-4]. Nevertheless, the gain/phase error, mutual coupling, and the sensor position error actually all have connections to the DOA, especially at high frequency [5], [6]. For the one-dimensional (1D) linear array, which is usually applied in the automotive radar [7], its array error depends not only on the azimuth but also on the elevation, even though it cannot estimate the elevation of a signal. Therefore, for high accuracy DOA estimation, 2D array error calibration is necessary. Another noteworthy problem is that usually, the array is protected by a cover/radome, which will deteriorate the array response further. Thus, we need to adopt offline calibration instead of online calibration.

There are mainly three signal processing-based offline calibration methods, i.e., the exhaustive search method, the gain/phase compensation method [8], and the array interpolation method [9], [10], which have been reviewed and analyzed in detail in Sec. 2. The conclusion is that these signal processing-based methods are not able to deliver satisfactory performance when the array error depends highly on the DOA of a signal.

With the development of machine learning, lots of data-driven methods have been proposed for DOA estimation [11-20], and some of them focus on handling the array errors [14-18]. In [18], a hierarchical framework of deep NNs is proposed to deal with the general DOA estimation problem, and the robustness with respect to array imperfections has been shown. In [14], a novel multi-frame phase 
enhancement method based on the long short-term memory (LSTM) NNs has been proposed for the reconstruction of a less distorted sample covariance matrix, which helps reduce the impact of array errors. However, the above two methods have only been verified by simulated data. In [17], the measured data obtained by a rectangular array is used for training an NN model for DOA estimation. In [16], a support vector regression (SVR)-based method is proposed for DOA estimation for a practical wide-beam highfrequency radar. Both the methods in [17] and [16] have shown improved performance over the conventional signal processing-based methods. Nevertheless, in the two methods, the training and testing data are on the same noise level. That is to say that the generalization capability regarding the SNR has not been considered. In [15], multiple machine learning-based methods for DOA estimation have been proposed, and they show good performance on testing data of different noise levels. However, the models in [15] have to been trained by data comprising samples of different noise levels. Besides, in the above methods, no method for augmenting the training data is given.

In this paper, we propose a new NN-based method for the $2 \mathrm{D}$ calibration of angularly dependent array error. The basic idea is to utilize the advantage of NN in approximating complex nonlinear functions. In this case, the function's input is defined to be the array output data, and the function's output is defined to be the DOA of a signal. For the construction of the training data, the array steering vectors are measured on an azimuth grid at different elevations in the anechoic chamber, and the off-grid steering vectors are derived via the proposed LMI technique to reduce overfitting risk. The phase differences in complex model are extracted from the steering vectors to form the input features. In order to make the method generalize well to the noisy data, we propose to train the model at the lowest SNR of application, which means our training data only comprise samples of the same noise level. In the end, the effectivity of the proposed method is tested by performing experiments on the measured data of a $77 \mathrm{GHz}$ automotive radar.

The rest of this paper is organized as follows. In Sec. 2, the model for array error calibration and the conventional signal processing-based calibration methods are introduced. In Sec. 3, the method of 2D angularly dependent array error calibration is described in detail, which consists of data augmentation via LMI, feature selection, model generalization to noisy data, and $\mathrm{NN}$ construction. The experimental evaluations are performed in Sec. 4 . Finally, Section 5 concludes the paper.

\section{Problem Formulation and Conventional Solutions}

Consider a linear array with $M$ sensors (or antennas) exposed to $K$ far-field narrowband signal sources. The 2D DOAs of the signals are denoted by $[\theta, \varphi]$ where $\boldsymbol{\theta}=\left[\theta_{1}, \ldots, \theta_{K}\right]$ is the azimuth vector and $\boldsymbol{\varphi}=\left[\varphi_{1}, \ldots, \varphi_{K}\right]$ is the elevation vector. The positions of the sensors are represented by $\boldsymbol{d}=\left[d_{1}, \ldots, d_{M}\right]$. If the array is assumed to be perfect, we can model the output of array as [21]

$$
\begin{aligned}
\boldsymbol{x} & =A(\boldsymbol{\theta}, \boldsymbol{\varphi}, \boldsymbol{d}) \boldsymbol{s}+\boldsymbol{\varepsilon} \\
& =A(\boldsymbol{\theta}, \boldsymbol{d}) \boldsymbol{s}+\boldsymbol{\varepsilon}
\end{aligned}
$$

where $\boldsymbol{A}(\boldsymbol{\theta}, \boldsymbol{d})$ is the $M$-by- $K$ array manifold matrix that has a well-known analytical expression with respect to $\theta, \boldsymbol{d}$ and is unrelated to $\varphi$ due to the perfect array assumption. $\boldsymbol{s}$ is the vector of baseband signals, and $\varepsilon$ is the zero-mean Gaussian noise vector that is temporally and spatially white.

In practice, the array error usually exists and can mainly be divided into three categories, i.e., gain/phase error, mutual coupling, and sensor position error. For the simplification of the calibration processing, these array errors are assumed to be independent of the DOA, which leads to the following modified array model [22], [23]

$$
\boldsymbol{x}=\boldsymbol{C} \boldsymbol{\Gamma} \boldsymbol{A}(\boldsymbol{\theta}, \boldsymbol{d}+\Delta d) \boldsymbol{s}+\varepsilon
$$

where the Toeplitz matrix $\boldsymbol{C}$ models the mutual coupling, the diagonal matrix $\Gamma$ models the gain/phase error, and $\Delta d$ models the sensor position error. On the basis of (2), online calibration, which jointly estimates the DOAs and calibration parameters, can be performed [2-4].

However, the angular independence assumption does not always coincide with the truth. Furthermore, in many cases, the array will be protected by a cover/radome, which will further deteriorate the uniformity of array error over different DOAs. We verify the phenomenon from the measurement data of the array of a $77 \mathrm{GHz}$ automotive radar. Since the field of interest (FOV) in azimuth is much larger than the FOV in elevation for the automotive radar, the array outputs are collected while a corner reflector is scanned over azimuth grid $-40^{\circ}$ to $40^{\circ}$ at elevations from $-3^{\circ}$ to $3^{\circ}$ in front of the radar in a millimeter anechoic chamber. In Fig. 1, the phase difference of the antennas with interspacing of $1.5 \lambda$ is plotted, where $\lambda$ is the wavelength. In this figure, "ele" means "elevation", "wr" means "with radome", and "wor" means "without radome". As shown in Fig. 1, firstly, at the elevation of $0^{\circ}$ with the radome, the phase difference experiences a larger angularly dependent nonlinear array error. Secondly, at different elevations with the radome, the phase difference is also influenced by different array errors (The difference of phase difference at different elevations can be larger than $20^{\circ}$ ). Thus, we can conclude that, in the practical application, the array error depends not only on the azimuth but also on the elevation.

To cope with the angularly dependent array error, we need to adopt the offline calibration instead of online calibration. Here, we make another assumption that there is only one source (i.e., $K=1$ ). This assumption is reasonable since the multiple sources can be separated in the time/frequency/Doppler domain in advance at high probability [24]. 


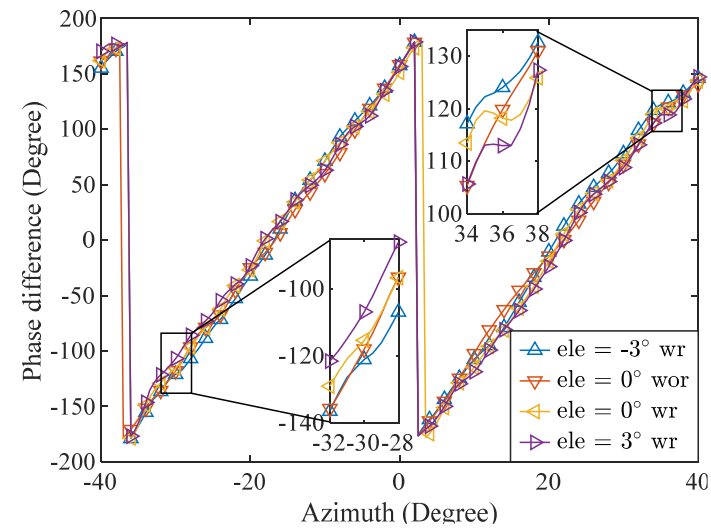

Fig. 1. Measured phase difference between antennas of a $77 \mathrm{GHz}$ automotive radar at different elevations without and with a radome.

For instance, the automotive radar with large bandwidth and large observation time can separate multiple sources in the range-Doppler domain. Under this assumption, the array model can be modified as

$$
\boldsymbol{x}=\boldsymbol{f}_{\varphi}(\theta) s+\boldsymbol{\varepsilon}
$$

where $s$ is the baseband signal, and $\boldsymbol{f}_{\varphi}(\theta)$ is the unknown array steering vector, which is also related to elevation $\varphi$. The offline calibration first measures the array steering vector $\boldsymbol{f}_{\varphi}(\theta)$ on the azimuth grid with interval of $\Delta \theta$ at different elevations. This can be done by scanning a corner reflector within the radar $2 \mathrm{D}$ FOV in the millimeter chamber. Since we can set a very high SNR, the measured array steering vectors can be derived as

$$
\hat{\boldsymbol{f}}_{i}(\theta)=\boldsymbol{x}_{\theta, \varphi_{i}} / s, \quad \theta \in \boldsymbol{\Omega}, i=1,2, \ldots, I
$$

where $\boldsymbol{x}_{\theta, \varphi_{i}}$ is the array output data when the corner reflector is placed at azimuth $\theta$, elevation $\varphi_{i}$. $\Omega$ is the azimuth grid within azimuth FOV, and the number of points in $\Omega$ is $L$. $I$ is the number of sampled elevations. From (4), we can find that for the measured array steering vector, we do not explicitly show its relation with the elevation but regard $\hat{f}_{i}(\theta), i=1,2, \ldots, I$ as the multiple noisy observations at azimuth $\theta$.

After the measured array steering vectors are obtained, the DOA estimation can be performed in the following three ways.

(1) Exhaustive search

Exhaustive search is to scan each measured steering vector to minimize or maximize the cost function of DOA estimation. For example, the beamforming-based exhaustive search method can be represented by [25]

$$
\hat{\theta}=\arg \max _{\substack{\theta \in \boldsymbol{\Omega},, i=1,2, \ldots, I}} \hat{\boldsymbol{f}}_{i}^{\mathrm{H}}(\theta) \boldsymbol{x} \boldsymbol{x}^{\mathrm{H}} \hat{\boldsymbol{f}}_{i}(\theta) .
$$

\section{(2) Gain/phase compensation}

Normally, the gain/phase compensation method only needs to measure the array steering vector at $\theta=\varphi=0^{\circ}$, which is represented by $\hat{\boldsymbol{f}}_{\varphi=0^{\circ}}\left(\theta=0^{\circ}\right)$. Then, before DOA estimation, the sampled data $\boldsymbol{x}$ needs to be calibrated by $\hat{\boldsymbol{f}}_{\varphi=0^{\circ}}\left(\theta=0^{\circ}\right)$, which can be written as

$$
\hat{\boldsymbol{x}}=\operatorname{diag}\left[1 / \hat{\boldsymbol{f}}_{\varphi=0^{\circ}}\left(\theta=0^{\circ}\right)\right] \boldsymbol{x}
$$

where $\operatorname{diag}(\cdot)$ is the operator to form a diagonal matrix with entries of a vector. $\hat{\boldsymbol{x}}$ is the calibrated sampled data, which can be fed into the DOA estimator. For example, if the conventional beamforming $(\mathrm{CBF})$ is chosen as the DOA estimator, then the DOA will be estimated as

$$
\hat{\theta}=\arg \max _{\theta} \boldsymbol{a}^{\mathrm{H}}(\theta) \hat{\boldsymbol{x}} \hat{\boldsymbol{x}}^{\mathrm{H}} \boldsymbol{a}(\theta)
$$

where $\boldsymbol{a}(\theta)=\left[a_{1}(\theta), a_{2}(\theta), \ldots, a_{M}(\theta)\right]^{\mathrm{T}}$ is the perfect array steering vector, and $a_{m}(\theta)=\exp \left(\mathrm{j} 2 \pi d_{m} \sin (\theta) / \lambda\right)$.

\section{(3) Array interpolation}

The array interpolation method calibrates the array data using all measured steering vectors within the FOV, and it can reduce the memory for storing the calibration information. For the $2 \mathrm{D}$ array error calibration, the FOV will also be 2D. It maps the measured steering vectors to the ideal steering vectors using the linear least squares (LS) method. The interpolating matrix can be achieved by solving the following linear optimization problem [8], [26]

$$
\hat{\boldsymbol{G}}=\arg \min _{\boldsymbol{G}} \sum_{\substack{\theta \in \boldsymbol{\Omega}, i=1,2, \ldots, I}}\left\|\boldsymbol{a}(\theta)-\boldsymbol{G} \hat{\boldsymbol{f}}_{i}(\theta)\right\|_{2}^{2}
$$

where $\|\cdot\|_{2}$ means the $\ell 2$ norm. We can find the calibration information is compressed into a smaller dimension matrix $\hat{\boldsymbol{G}}$. Then, before array data $\boldsymbol{x}$ is fed into DOA estimator, it needs to be calibrated by $\hat{\boldsymbol{x}}=\hat{\boldsymbol{G}} \boldsymbol{x}$.

The exhaustive search method can ensure the DOA estimation of on-grid sources not be affected by the array error and do interpolation for the off-grid sources. However, it needs high calculation power to support the exhaustive search and large memory to store the measured steering vectors. For the gain/phase compensation method, it only needs to measure and store one steering vector, and the computational complexity can be very low (e.g., DOA estimation can be performed by the interferometer method [27]). Nevertheless, the DOA estimation of the sources that are not at $\theta=\varphi=0^{\circ}$ will be largely affected by the array error. For the array interpolation method, only a small matrix $\hat{\boldsymbol{G}}$ needs to be stored, and the calibration performance is usually better than the gain/phase compensation method. However, due to the angular dependence of the array error, the linear minimization of problem (8) will have a large residual error, which leads to insufficient calibration.

\section{Angularly Dependent Array Error Calibration via Neural Network}

From (3) we know, with angularly dependent array error, $\boldsymbol{x} \mapsto \theta$ represents a highly nonlinear unknown 
function. Performing DOA estimation is equivalent to finding this function, which is not an easy problem for the conventional signal processing-based methods described in the last section. Fortunately, as one of the machine learning methods, NN is good at nonlinear function modelling [28]. Provided with a number of function inputs and outputs, i.e., the training set, the $\mathrm{NN}$ can be fitted to approximate the function relationship, the procedure of which is called $\mathrm{NN}$ training.

Since the output of the function is a continuous variable that has a wide range, we use the $\mathrm{NN}$ to perform the regression task. For the array calibration case, to do the training, we need to first solve three problems, which are data augmentation, feature selection, and model generalization to noisy data. We describe them as follows.

\subsection{Data Augmentation by Local Manifold Interpolation}

The steering vectors $\hat{f}_{i}(\theta), i=1,2, \ldots, I$ are measured on azimuth grid $\boldsymbol{\Omega}$ at different elevations in the anechoic chamber. For the reduction of time consumption of the offline calibration during mass production, the azimuth grid cannot be too dense. Otherwise, it will take too much time to collect the calibration data. This restriction, on the one hand, will lead to a small number of training samples and overfitting of the network during training, and on the other hand, will make the trained model generalize poorly to the off-grid sources.

We propose the LMI technique to augment the training samples. Assume $\theta_{l}, \theta_{l+1} \in \boldsymbol{\Omega}$ are two adjacent grid points for measuring the steering vectors. For the off-grid angle $\theta_{l}<\theta<\theta_{l+1}$, we can interpolate its off-grid steering vector using the measured steering vectors on its $M^{\prime}$ nearby grid points, where $M^{\prime}$ is the number of rows of the local manifold matrix. We call this method the LMI. This is different from the interpolation method in (8) called the global manifold interpolation that uses all measured steering vectors. The LMI is more reasonable since the curve of array response normally is smooth, i.e., the steering vectors within a small angle interval will not change much. To further augment data size and increase robustness, for each $\theta$, we can interpolate $M^{\prime}-1$ steering vectors using $M^{\prime}-1$ different sets of the adjacent continuous measured steering vectors. For example, if $M^{\prime}=4$, for each $\theta_{l}<\theta<\theta_{l+1}$, we can choose the three sets of local azimuth grid represented by $\left[\theta_{l-2}, \theta_{l-1}, \theta_{l}, \theta_{l+1}\right], \quad\left[\theta_{l-1}, \theta_{l}, \theta_{l+1}, \theta_{l+2}\right], \quad$ and $\left[\theta_{l}, \theta_{l+1}, \theta_{l+2}, \theta_{l+3}\right]$. For each set of the local azimuth grid which is represented by $\Omega^{\prime}$, the corresponding local interpolating matrix can be obtained by solving the following LS problem

$$
\begin{aligned}
\hat{\boldsymbol{G}}_{i}^{\prime} & =\arg \min _{\boldsymbol{G}^{\prime}} \sum_{\theta^{\prime} \in \boldsymbol{\Omega}^{\prime}}\left\|\hat{\boldsymbol{f}}_{i}\left(\theta^{\prime}\right)-\boldsymbol{G}^{\prime} \boldsymbol{a}\left(\theta^{\prime}\right)\right\|_{2}^{2} \\
& =\hat{\boldsymbol{F}}_{i}\left(\boldsymbol{\Omega}^{\prime}\right) \boldsymbol{A}^{+}\left(\boldsymbol{\Omega}^{\prime}\right)
\end{aligned}
$$

where $(\cdot)^{+}$means the pseudoinverse. $\hat{\boldsymbol{F}}_{i}\left(\boldsymbol{\Omega}^{\prime}\right)$ and $\boldsymbol{A}\left(\boldsymbol{\Omega}^{\prime}\right)$ are the local manifold matrices whose columns are composed of the measured steering vectors and the perfect steering vectors on the local azimuth grid $\boldsymbol{\Omega}^{\prime}$, respectively. Then the interpolated steering vector can be achieved by

$$
\hat{\boldsymbol{f}}_{i}(\theta)=\hat{\boldsymbol{G}}_{i}^{\prime} \boldsymbol{a}(\theta) \text {. }
$$

Assume $P$ off-grid steering vectors need to be interpolated in each interval between two adjacent grid points. According to the data augmentation method, the number of samples after augmentation will be $\left(L+\left(L-M^{\prime}+1\right)\left(M^{\prime}-1\right) P\right) I$.

\subsection{Feature Selection}

Since the output is the DOA $\theta$ and we have assumed there is only one source, according to the interferometer method, the relation between the DOA and phase difference can be written as [27]

$$
\phi=2 \pi d \sin (\theta) / \lambda
$$

where $\phi$ is the phase difference between the sensors with interspacing of $d$. We can observe that, in theory, the DOA is only related to the phase difference. Also, note that when $d>\lambda / 2$, phase wrapping happens on $\phi[29]$. Thus, we choose the feature as follows

$$
\gamma=\exp (\mathrm{j} \phi)=\exp (\mathrm{j} 2 \pi d \sin (\theta) / \lambda)
$$

where we call $\gamma$ the phase difference in complex mode. Another advantage of (12) is the $\gamma$ calculated from $\hat{\boldsymbol{f}}_{i}(\theta)$ and from $\boldsymbol{x}_{\theta, \varphi_{i}}$ are the same, which is to say that just using the previously measured $\hat{f}_{i}(\theta)$, we are able to train the NN.

For an $M$-element linear array, we can extract $N=C_{M}^{2}=M(M-1) / 2$ pairs of sensors with nonzero interspacing. Thus, we can get $N$ features, which are $\gamma_{1}, \gamma_{2}, \ldots, \gamma_{N}$. At last, due to the fact that $\mathrm{NN}$ can only deal with the real number, we split the real and imaginary parts of the complex number, leading to $2 N=M(M-1)$ features in total.

\subsection{Model Generalization to Noisy Data}

To make the NN for array calibration practical, we need to ensure it has good prediction performance for the noisy data. However, for now, we only have the array steering vectors measured at very high SNR. If the model is trained based on these high SNR data, then it will generalize poorly to the low SNR data [30] since the model has no prior information about these noisy data.

Our strategy for this is to train the model based on the steering vectors measured under the lowest SNR within the SNR dynamic range of the application. To explain the reasonability, we write (3) in low SNR and high SNR mode

$$
\left\{\begin{array}{l}
\boldsymbol{x}_{\mathrm{Lo}}=\boldsymbol{f}_{\varphi}(\theta) s+\boldsymbol{\varepsilon}_{\mathrm{Lo}} \\
\boldsymbol{x}_{\mathrm{Hi}}=\boldsymbol{f}_{\varphi}(\theta) s+\boldsymbol{\varepsilon}_{\mathrm{Hi}}
\end{array}\right.
$$


where the subscript 'Lo' and 'Hi' represent low SNR and high SNR, respectively. Since $\varepsilon_{\mathrm{Lo}}$ and $\varepsilon_{\mathrm{Hi}}$ are both the Gaussian noise, if we generate as many $\varepsilon_{\mathrm{Lo}}$ as possible via the Monte-Carlo method, then the generated low SNR data will cover the high SNR data, and the model trained on the low SNR data will be expected to generalize well to the high SNR data.

As the calibration data is measured under very high SNR, we can regard it as noise-free data. Then we add zero-mean Gaussian noise $\varepsilon$ to the noise-free data to generate data at the lowest SNR of application. After that, the steering vector is calculated by (4) again. For each aforementioned sample, we use Monte-Carlo method to generate $Q$ noisy samples (i.e., $\varepsilon$ is realized $Q$ times). Then the final number of training samples is $(L+(L-M+1)(M-1) P) I Q$.

\subsection{Construction of the Neural Network}

After the training samples are generated, we can construct the NN. We adopt the fully-connected $\mathrm{NN}$ architecture with $G$ cascaded layers. Except for the input layer and the output layer, the other $G-2$ layers in the middle are referred to as the hidden layers. Define the first hidden layer as layer 1. Accordingly, the output layer will be layer $G-1$. Since each training sample has $2 N$ features, we use $z \in \mathbb{R}^{2 N \times 1}$ to represent an input of the NN. The output of the NN can be written as

$$
\hat{\theta}=\mathrm{NN}(\boldsymbol{z} ; \boldsymbol{\Lambda})=\boldsymbol{f}^{(G-1)}\left(\cdots \boldsymbol{f}^{(2)}\left(\boldsymbol{f}^{(1)}(\boldsymbol{z})\right)\right)
$$

where $\boldsymbol{\Lambda}=\left\{\boldsymbol{W}^{(g)}, \boldsymbol{b}^{(g)}\right\}_{g=1}^{G-1}$ is the set of unknown network parameters. $f^{g(g)}(\cdot)$ is the transformation function of the layer $g$, which can be represented as

$$
\boldsymbol{f}^{(g)}\left(\boldsymbol{z}^{(g)}\right)=\boldsymbol{h}^{(g)}\left(\boldsymbol{W}^{(g)} \boldsymbol{z}^{(g)}+\boldsymbol{b}^{(g)}\right), g=1,2, \ldots, G-1
$$

where $\boldsymbol{z}^{(g)}, \boldsymbol{W}^{(g)}, \boldsymbol{b}^{(g)}, \boldsymbol{h}^{(g)}(\cdot)$ are the input, the weight matrix, the bias, and the activation function of layer $g$, respectively. Moreover, we have $\boldsymbol{z}^{(1)}=\boldsymbol{z}$.

For each training sample, we have a $\boldsymbol{z}$ and its known label $\theta$. Training the $\mathrm{NN}$ is equivalent to estimating $\boldsymbol{\Lambda}$ via minimizing the following cost function

$$
\operatorname{Loss}(\boldsymbol{\Lambda})=\sum\|\theta-\hat{\theta}\|_{2}^{2}
$$

where the summation is performed over the samples. After optimal $\boldsymbol{\Lambda}$ is obtained, we can predict the label of the input by using (14)

Finally, we conclude the proposed method in Fig. 2. On the left is the training part. The data measured in the anechoic chamber are used to obtain the measured array steering vectors. In this step, we augment the data by LMI and generate noisy data. Then the measured steering vectors are used to generate the training features, which are the phase differences in the complex mode. After training the

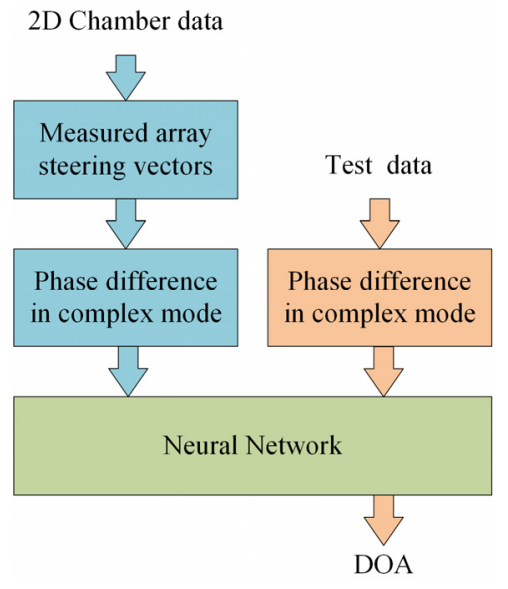

Fig. 2. Block diagram of neural network-based array calibration method.

$\mathrm{NN}$, we will test it using the test data that has not been seen by the model and output the predicted DOA.

\section{Experimental Evaluation}

In this section, we perform experiments to evaluate the performance of the proposed method. We use a $77 \mathrm{GHz}$ FMCW automotive radar with a 2T4R (two transmitters and four receivers) linear array with a radome. Using the MIMO processing, we effectively have $M=8$ sensors. Its azimuth FOV is from $-40^{\circ}$ to $40^{\circ}$ and elevation FOV is from $-3^{\circ}$ to $3^{\circ}$. In the millimeter chamber, we put a corner reflector in front of the radar, and scan it on azimuth grid $\left[-40^{\circ}, 40^{\circ}\right]$ with the interval of $0.5^{\circ}$ at elevations $-3^{\circ}$, $-2^{\circ}, \ldots, 3^{\circ}$. The measured data are extracted from the range-Doppler domain, and the SNR is about $60 \mathrm{~dB}$. The measured data on integer grid points (i.e., $\left[-40^{\circ},-39^{\circ}, \ldots\right.$, $\left.40^{\circ}\right]$ ) at elevations $-3^{\circ},-2^{\circ}, \ldots, 3^{\circ}$ are used to construct the calibration (training) data, and the measured data on decimal grid points (i.e., $\left[-39.5^{\circ},-38.5^{\circ}, \ldots, 39.5^{\circ}\right]$ ) at elevations $-3^{\circ},-2^{\circ}, \ldots, 3^{\circ}$ are used to test the calibration performance. Thus, we have $I=7$. The proposed $\mathrm{NN}$ is compared with the gain/phase compensation method and array interpolation method, which are abbreviated as "Gain/phase" and "Interpolation", respectively. The DOA estimators of the signal processing-based methods are chosen as the CBF.

During the step of constructing the training data, when adopting the LMI, we have $L=81, P=9$. Due to the MIMO architecture, the 8-element array can be split into two 4-element arrays, which can be interpolated independently. So, we have $M^{\prime}=4$. When generating the noisy samples, we set $Q=100$. Therefore, the total number of the noisy training samples will be $\left(L+\left(L-M^{\prime}+1\right)\left(M^{\prime}-1\right) P\right) I Q=1530900$. For the feature selection, from the measured steering vector, we extract $N=M(M-1) / 2=28$ phase differences in complex mode and finally get 56 features after real and imaginary number splitting. The NN is trained using MATLAB software and the training parameters are listed in Tab. 1. 


\begin{tabular}{|c|c|}
\hline Parameter name & Value \\
\hline Size of training samples & $56 \times 1530900$ \\
\hline Number of hidden layers & 5 \\
\hline Number of neurons on each layer & 32 \\
\hline Type of activation function & ReLU \\
\hline Type of solver & Adam \\
\hline Max epochs & 1000 \\
\hline Batch size & 14336 \\
\hline Initial learning rate & 0.001 \\
\hline$\ell 2$ regularization & 0.0001 \\
\hline
\end{tabular}

Tab. 1. The training parameters of the neural network.

\subsection{Calibration Performance under Noise- Free Condition}

Here noise-free condition means we do not manually add noise to the chamber data and test data. So, in this case, the number of training samples is 15309 , and when training the $\mathrm{NN}$, we decrease the batch size to 896 . The noise-free calibration performances are reflected by the DOA estimation error evaluated on the test data at elevation $0^{\circ}$, which is shown in Fig. 3, and by the root-meansquare errors (RMSEs) of DOA estimation at different elevations, which are shown in Fig. 4. Here we also include the result of the $\mathrm{NN}$ trained only by the data at elevation $0^{\circ}$.

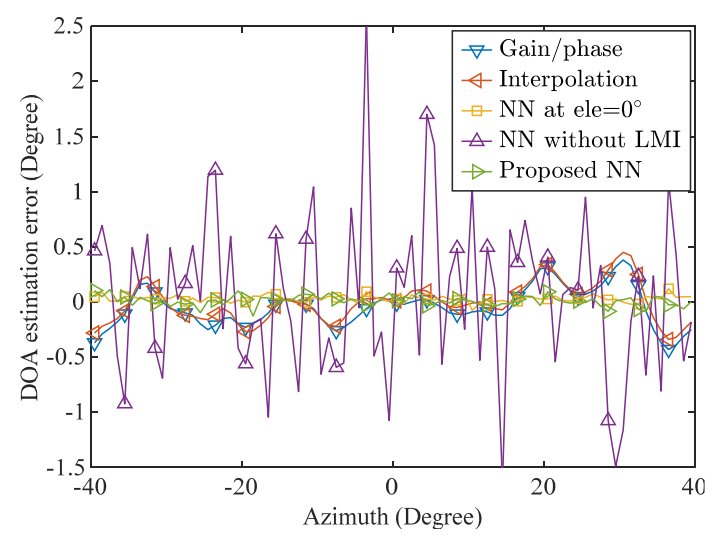

Fig. 3. Comparison of DOA estimation error evaluated on noise-free test data at elevation $0^{\circ}$ after calibration.

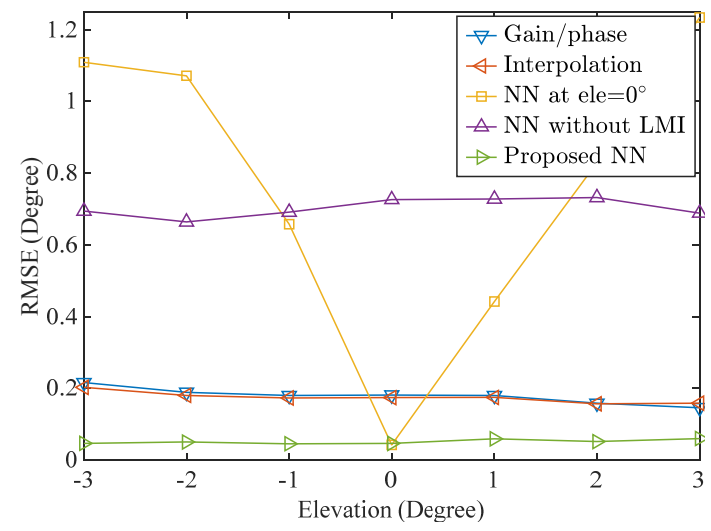

Fig. 4. RMSE comparison of DOA estimation evaluated on noise-free test data at different elevations after calibration.
The RMSE of DOA estimation at the $i$-th elevation is calculated as

$$
R M S E_{i}=\sqrt{\left\|\boldsymbol{\theta}_{i}-\hat{\boldsymbol{\theta}}_{i}\right\|_{2}^{2} /\left|\boldsymbol{\theta}_{i}\right|}
$$

where $|\cdot|$ takes the length of a vector, and the vectors $\boldsymbol{\theta}_{i}$ and $\hat{\boldsymbol{\theta}}_{i}$ are composed of the true and estimated DOAs of the sources on the azimuth test grid at the $i$-th elevation, respectively.

From Fig. 3 and Fig. 4, we can find that if the NN is trained only at elevation $0^{\circ}$, then it will have the best calibration performance at elevation $0^{\circ}$ but will have the worst performance at large elevations. If the LMI is not adopted in the proposed NN method, then the calibration performance will deteriorate due to the overfitting of the $\mathrm{NN}$ model caused by a smaller training set. If the $\mathrm{NN}$ is trained at multiple elevations, it will have good performance in the whole $2 \mathrm{D}$ FOV. The array interpolation method calibrates the array a little better than the gain/phase compensation, and the calibration performance of the proposed NN-based method surpasses the other two signal processing-based methods, achieving the DOA estimation error about less than $0.1^{\circ}$.

\subsection{Calibration Performance under Noisy Condition}

Here we compare the statistical calibration performances of network models trained with noisy data at different SNRs. The statistical calibration performance is also reflected by the RMSE of the DOA estimation that is calculated as

$$
R M S E_{\text {noisy }}=\sqrt{\sum_{j=1}^{J} \sum_{i=1}^{I} \frac{\left\|\boldsymbol{\theta}_{i}-\hat{\boldsymbol{\theta}}_{i}^{j}\right\|_{2}^{2}}{\left|\boldsymbol{\theta}_{i}\right| I J}}
$$

where $J$ is the number of Monte-Carlo experiments, which is set as $J=500$, and $\hat{\boldsymbol{\theta}}_{i}^{j}$ is the vector composed of the estimated DOAs on the azimuth test grid at the $i$-th elevation in the $j$-th experiment. The SNR is varied from $15 \mathrm{~dB}$ to $50 \mathrm{~dB}$. Here we only care about the performance above $15 \mathrm{~dB}$ because $15 \mathrm{~dB}$ is often the SNR detection threshold of most systems. For performance reference, the DOA estimation performance of the perfect array with the same geometry is included.

From the comparison results shown in Fig. 5, we can find that the RMSE of the array interpolation method is a little lower than that of the gain/phase compensation method, and their RMSEs keep almost constant under different SNRs due to large residual array error. Moreover, the model trained at high SNR generalizes poorly for the low SNR data. However, if the NN is trained at the lowest SNR (i.e., $15 \mathrm{~dB}$ ), the calibration performance will surpass the signal processing-based methods under all SNRs (by at least $55 \%$ on average in RMSE) and will be close to the 


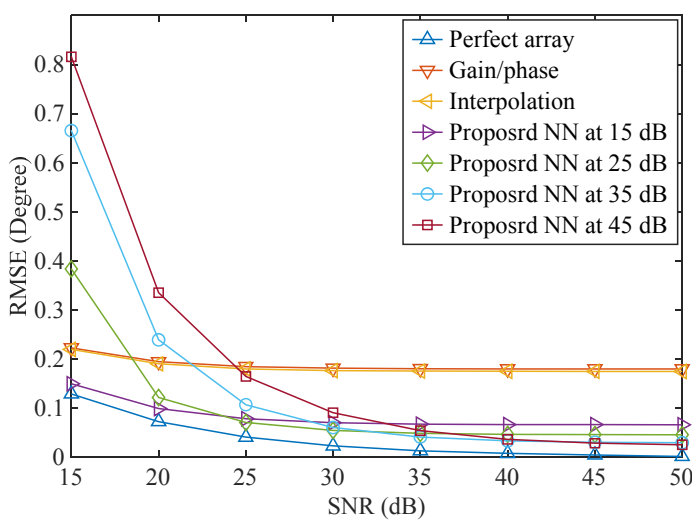

Fig. 5. RMSE comparison of DOA estimation on noisy test data versus SNR after calibration.

perfect array without array error under low to medium SNR. Besides, the model trained at the lowest SNR has a slightly higher RMSE than the models trained at high SNR. However, this is not a problem in the practical application. Due to the fact that the model trained at only one SNR point has satisfactory performance over the entire SNR range, there is no need to train the model for a range of SNR, which helps save the computational resource.

\subsection{Performance Verification using Outdoor Road Measurement}

In the last experiment, we will use the practical outdoor road measurement data to verify the proposed NNbased calibration method. This is important because we need to confirm whether the model trained on the indoor measured data mixed with the Gaussian noise works in the practical outdoor noisy situation. The road measurement scenario is shown in Fig. 6, where we can see two cars and a truck on the road. On the right side of the road, we also can see a line of lamps and the guardrail.

The data collected by the radar is processed by $2 \mathrm{D}$ FFT, constant false alarm rate (CFAR) detection, and then the array data is processed by the gain/phase compensation, array interpolation, and the proposed NN trained at $15 \mathrm{~dB}$. The estimated DOAs and the ranges of the targets are used to map the targets on the $\mathrm{x}-\mathrm{y}$ plane shown in Fig. 7. In Fig. 7, we have not performed the clustering operation. So we can observe multiple points corresponding to a target due to the multiple scatters on a target. From the results of the

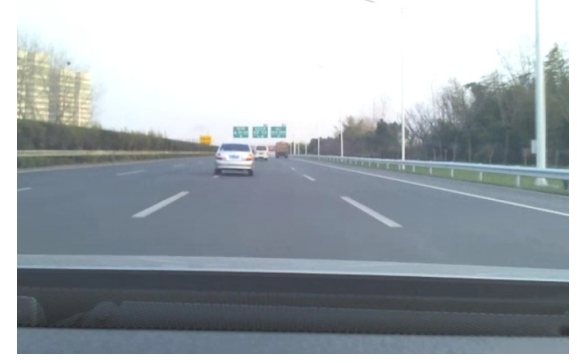

Fig. 6. The road measurement scenario.

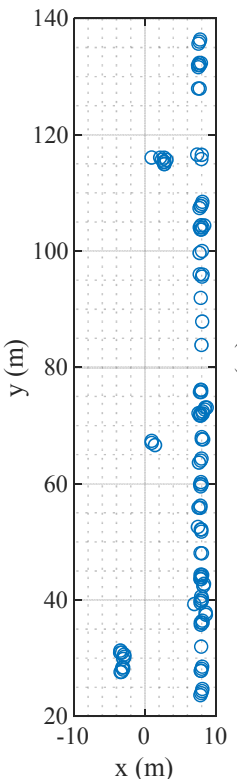

(a)

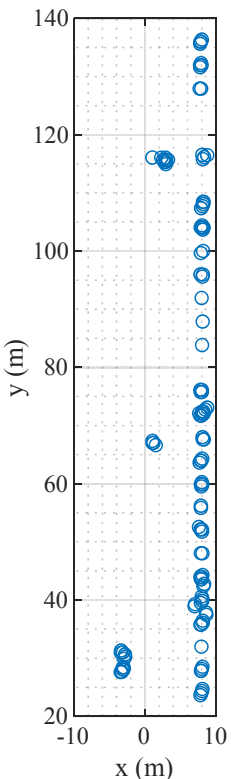

(b)

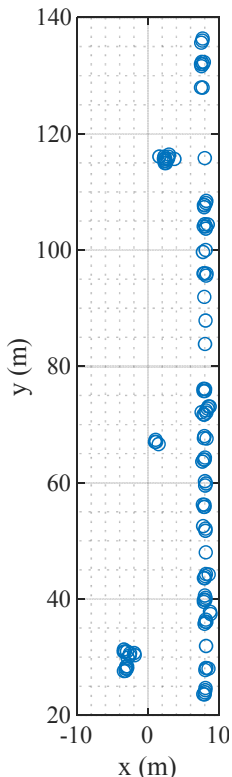

(c)
Fig. 7. The estimated positionings of targets before clustering operation: (a) Gain/phase compensation, (b) array interpolation, (c) the proposed $\mathrm{NN}$ at $15 \mathrm{~dB}$.

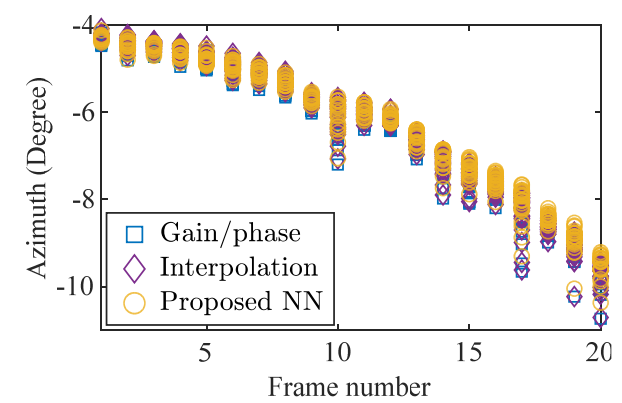

(a)

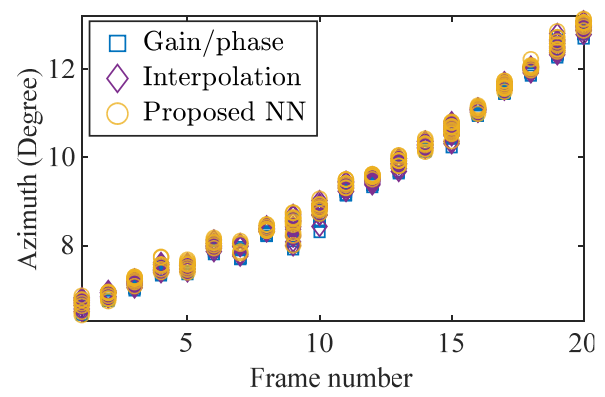

(b)

Fig. 8. Comparison of estimated DOAs over multiple continuous frames from different methods: (a) A target with negative azimuth, (b) a target with positive azimuth.

signal processing-based methods and the NN-based method, we can clearly see three targets corresponding to the vehicles and a line of barriers corresponding to the lamps and the guardrail. This indicates our proposed NNbased calibration method can work in the practical outdoor situation. Besides, in Fig. 7, we can observe some differences between the NN-based methods and the signal processing-based methods, although the differences are not significant due to the large-scale scenario. 
Besides, we also extract the estimated DOAs related to one same target over multiple continuous frames. The results are shown in Fig. 8. In Fig. 8(a), the scatters of the target have negative azimuths. We can find the estimated DOAs of the signal processing-based methods start to deviate from those of the proposed NN method significantly when the azimuth is around $-8^{\circ}$, which is consistent with the noiseless DOA estimation performance shown in Fig. 3. In Fig. 8(b), the scatters have positive azimuth, and the deviation is smaller than that in Fig. 8(a), which can be explained by the fact that around azimuth of $10^{\circ}$, the performance difference between the signal processing-based methods and the proposed NN method shown in Fig. 3 is small. Thus, the last experiment also confirms that the results of outdoor measurements are consistent with those of indoor measurements.

\section{Conclusion}

In this paper, we proposed a new NN-based method for calibrating the 2D angularly dependent array error. In this method, the measured calibration data is augmented by the proposed LMI technique to avoid overfitting of the NN model, and Gaussian noise is added into the calibration data to improve the generalization performance to the noisy data. The performance of the proposed method is verified by the indoor and outdoor measured data from a $77 \mathrm{GHz}$ automotive radar. It shows the calibration performance of $\mathrm{NN}$ trained at the lowest SNR surpasses the conventional signal processing methods largely under all SNRs and is close to the performance of the perfect array without array error under low to medium SNR. Since the model trained at the lowest SNR works well for a large range of SNR, there is no need to do training for a range of SNR.

\section{Acknowledgments}

This work was supported by the National Natural Science Foundation of China under Grant number 61701145; the Open Research Program of State Key Laboratory of Millimeter Waves under Grant number K202034.

\section{References}

[1] VAN TREES, H. L. Optimum Array Processing: Part IV of Detection, Estimation, and Modulation Theory. New York: John Wiley \& Sons, 2002. ISBN: 9780471093909

[2] FRIEDLANDER, B., WEISS, A. J. Direction finding in the presence of mutual coupling. IEEE Transactions on Antennas and Propagation, 1991, vol. 39, no. 3, p. 273-284. DOI: $10.1109 / 8.76322$

[3] YE, Z., LIU, C. On the resiliency of MUSIC direction finding against antenna sensor coupling. IEEE Transactions on Antennas and Propagation, 2008, vol. 56, no. 2, p. 371-380. DOI: 10.1109/TAP.2007.915461

[4] LIU, Z. M., ZHOU, Y. Y. A unified framework and sparse Bayesian perspective for direction-of-arrival estimation in the presence of array imperfections. IEEE Transactions on Signal Processing, 2013, vol. 61, no. 15, p. 3786-3798. DOI: 10.1109/TSP.2013.2262682

[5] FRIEDLANDER, B. Array calibration in the presence of linear manifold distortion. In Conference Record of 51st Asilomar Conference on Signals, Systems and Computers, ACSSC 2017. Pacific Grove (CA, USA), 2018, p. 1199-1203. DOI: 10.1109/ACSSC.2017.8335541

[6] FRIEDLANDER, B. Antenna array manifolds for high-resolution direction finding. IEEE Transactions on Signal Processing, 2018, vol. 66, no. 4, p. 923-932. DOI: 10.1109/TSP.2017.2778683

[7] SUN, S., PETROPULU, A. P. A sparse linear array approach in automotive radars using matrix completion. In Proceedings of the IEEE International Conference on Acoustics, Speech and Signal Processing (ICASSP). 2020, p. 8614-8618. DOI: 10.1109/ICASSP40776.2020.9053894

[8] TUNCER, T. E., FRIEDLANDER, B. (Eds.) Classical and Modern Direction-of-Arrival Estimation. Burlington: Academic Press, 2009. ISBN: 9780123745248

[9] HYBERG, P., JANSSON, M., OTTERSTEN, B. Array interpolation and DOA MSE reduction. IEEE Transactions on Signal Processing, 2005, vol. 53, no. 12, p. 4464-4471. DOI: 10.1109/TSP.2005.859341

[10] HYBERG, P., JANSSON, M., OTTERSTEN, B. Array interpolation and bias reduction. IEEE Transactions on Signal Processing, 2014, vol. 52, no. 10, p. 2711-2720. DOI: 10.1109/TSP.2004.834402

[11] CHAKRABARTY, S., HABETS, E. A. P. Multi-speaker DOA estimation using deep convolutional networks trained with noise signals. IEEE Journal of Selected Topics in Signal Processing, 2018, vol. 13, no. 1, p. 8-21. DOI: 10.1109/JSTSP.2019.2901664

[12] WU, L., LIU, Z. M., HUANG, Z. T. Deep convolution network for direction of arrival estimation with sparse prior. IEEE Signal Processing Letters, 2019, vol. 26, no. 11, p. 1688-1692. DOI: 10.1109/LSP.2019.2945115

[13] ELBIR, A. M. DeepMUSIC: Multiple signal classification via deep learning. IEEE Sensors Letters, 2019, vol. 4, no. 4, p. 1-4. DOI: $10.1109 /$ LSENS.2020.2980384

[14] XIANG, H., CHEN, B., YANG, M., et al. Improved direction-ofarrival estimation method based on LSTM neural networks with robustness to array imperfections. Applied Intelligence, 2021, vol. 51, p. 4420-4433. DOI: 10.1007/s10489-020-02124-1

[15] KHAN, A., WANG, S., ZHU, Z. Angle-of-arrival estimation using an adaptive machine learning framework. IEEE Communications Letters, 2019, vol. 23, no. 2, p. 294-297. DOI: 10.1109/LCOMM.2018.2884464

[16] WANG, R., WEN, B., HUANG, W. A support vector regressionbased method for target direction of arrival estimation from HF radar data. IEEE Geoscience and Remote Sensing Letters, 2018, vol. 15, no. 5, p. 674-678. DOI: 10.1109/LGRS.2018.2807405

[17] AGatonoviĆ, M., StankOViC, Z., MilOVANOVIC, I., et al. Efficient neural network approach for 2D DOA estimation based on antenna array measurements. Progress in Electromagnetics Research, 2013, vol. 137, p. 741-758. DOI: 10.2528/PIER13012114

[18] LIU, Z. M., ZHANG, C., YU, P. S. Direction-of-arrival estimation based on deep neural networks with robustness to array imperfections. IEEE Transactions on Antennas and Propagation, 
2018, vol. 66, no. 12, p. 7315-7327. DOI: 10.1109/TAP.2018.2874430

[19] GUO, Y., ZHANG, Z., HUANG, Y., et al. DOA estimation method based on cascaded neural network for two closely spaced sources. IEEE Signal Processing Letters, 2020, vol. 27, p. $570-574$. DOI: $10.1109 /$ LSP.2020.2984914

[20] HUANG, H., YANG, J., HUANG, H., et al. Deep learning for super-resolution channel estimation and DOA estimation based massive MIMO system. IEEE Transactions on Vehicular Technology, 2018, vol. 67, no. 9, p. 8549-8560. DOI: 10.1109/TVT.2018.2851783

[21] PAN, Y., LUO, G. Q., JIN, H., et al. DOA estimation with planar array via spatial finite rate of innovation reconstruction. Signal Processing, 2018, vol. 153, p. 47-57. DOI: 10.1016/j.sigpro.2018.07.001

[22] GUO, Y., ZHANG, Y., TONG, N., et al. Angle estimation and self-calibration method for bistatic MIMO radar with transmit and receive array errors. Circuits, Systems, and Signal Processing, 2017, vol. 36 , no. 4, p. 1514-1534. DOI: $10.1007 /$ s00034-016$0365-9$

[23] LIU, H., ZHAO, L., LI, Y., et al. A sparse-based approach for DOA estimation and array calibration in uniform linear array. IEEE Sensors Journal, 2016, vol. 16, no. 15, p. 6018-6027. DOI: 10.1109/JSEN.2016.2577712

[24] HUANG, C. J., DAI, C. W., TSAI, T. Y., et al. A closed-form phase-comparison ML DOA estimator for automotive radar with one single snapshot. IEICE Electronics Express, 2013, vol. 10, no. 7 , p. 1-7. DOI: $10.1587 /$ elex.10.20130086

[25] KRISHNAVENI, V., KESAVAMURTHY, T., APARNA, B. Beamforming for direction-of-arrival (DOA) estimation-a survey. International Journal of Computer Applications, 2013, vol. 61, no. 11, p. 4-11. DOI: 10.5120/9970-4758

[26] FRIEDLANDER, B. The root-MUSIC algorithm for direction finding with interpolated arrays. Signal Processing, 1993, vol. 30, no. 1, p. 15-29. DOI: 10.1016/0165-1684(93)90048-F

[27] PAN, Y., ZHANG, X. F., XIE, S. Y., et al. An ultra-fast DOA estimator with circular array interferometer using lookup table method. Radioengineering, 2015, vol. 24, no. 3, p. 850-856. DOI: $10.13164 /$ re.2015.0850

[28] Goodfellow, I., Bengio, Y., COURVIlle, A. Deep Learning. MIT Press, 2016. ISBN: 978-0262035613

[29] HANSSEN, R. F. Radar Interferometry: Data Interpretation and Error Analysis. Springer Netherlands, 2001. ISBN: 9780792369455, DOI: 10.1007/0-306-47633-9
[30] JIAN, T., RENDON, B. C., OJUBA, E., et al. Deep learning for $\mathrm{RF}$ fingerprinting: A massive experimental study. IEEE Internet of Things Magazine, 2020, vol. 3, no. 1, p. 50-57. DOI: 10.1109/iotm.0001.1900065

\section{About the Authors ...}

Yujian PAN (corresponding author) was born in 1987. He received his Ph.D. degree from the National University of Defense Technology in 2015 . He is currently an associated professor with the School of Electronics and Information, Hangzhou Dianzi University, Hangzhou, China. His main research interests include array signal processing, radar system design, radar system, and machine learning. From 2019 to 2020, he was a visiting scholar at the Department of Electrical Engineering (ESAT), KU Leuven, Belgium.

Sreeraj RAJENDRAN received the master's degree in Communication and Signal Processing from the Indian Institute of Technology, Bombay, India, in 2013, and the Ph.D. degree from KU Leuven, Belgium, in 2019, where he is a Post-Doctoral Researcher with the Networked Systems Group. In 2013, he was a Senior Design Engineer with the Baseband Team, Cadence (Tensilica). He was also an ASIC Verification Engineer with Wipro Technologies from 2007 to 2010. His main research interests include machine learning algorithms for wireless spectrum awareness and low power wireless sensor networks.

Sofie POLLIN obtained her Ph.D. degree at KU Leuven with honors in 2006. From 2006 to 2008 she continued her research on wireless communication, energy efficient networks, cross-layer design, coexistence and cognitive radio at UC Berkeley. In November 2008, she returned to IMEC to become a principal scientist in the green radio team. Since 2012, she is tenure track assistant professor at the Electrical Engineering Department at KU Leuven. Her research centers around networked systems that require networks that are ever more dense, heterogeneous, battery powered and spectrum constrained. Prof. Pollin is BAEF and Marie Curie fellow, and IEEE senior member. 